\title{
Puntos de vista sobre el 15-M. Aspectos pragmaestilísticos y deriva ideológica ${ }^{1}$
}

\author{
Adolf PIQUER VIDAL \\ Universidad Jaume I de Castelló \\ apiquer@uji.es
}

Recibido: $05 / 04 / 2012$

Aceptado: 18/12/2012

\begin{abstract}
Resumen
El presente estudio parte de la elección de un hecho de repercusión mediática con la intención de ofrecer una muestra, a través de los escritos en los periódicos del momento, de las implicaciones ideológicas que se derivan de determinadas selecciones lingüísticas en su uso pragmático y estilístico. La configuración del discurso de los periódicos alrededor del 15-M español ha servido como corpus de análisis para mostrar algunas cuestiones que, más allá del tema elegido, evidencian la inclusión de puntos de vista que contrastan de una manera notable según quién sea el emisor del mensaje, la oportunidad que brinde la cronología de los hechos y la conveniencia de un enfoque determinado según se fueron desarrollando los acontecimientos.
\end{abstract}

Palabras clave: $15-\mathrm{M}$, pragmaestilística, retórica, política, punto de vista.

\section{Views on Spanish 15-M. Pragma-stylistic Aspects and Ideological Tendences}

\begin{abstract}
This paper departs from a choice made with the intention of offering a sample, through the writings in newspapers around the end of May 2011, how to use various rhetorical, pragmatic and stylistic devices in the ideological orientation. The configuration of the speech around the 15-M Spanish movement has served as a corpus to test and show some issues beyond the chosen subject, show the contrasting views in a remarkable way according to who's the sender of message, the chance offered by the chronology of events and convenience of a particular approach as events were developing.
\end{abstract}

Keywords: 15-M, pragma-stylistic, rhetoric, politics, point of view.

\section{Referencia normalizada}

PIQUER VIDAL, Adolf (2013): "Puntos de vista sobre el 15-M. Aspectos pragmaestilísticos y deriva ideológica". Estudios sobre el mensaje periodístico. Vol. 19, Núm. 1, págs.: 223-234. Madrid, Servicio de Publicaciones de la Universidad Complutense.

Sumario: 1. Más allá de los géneros de opinión. 2. Lo dicho y su interpretación, o el malicioso uso de las comillas. 3. Dimensión pragmaestilística del discurso. 4. Conclusión: estrategias discursivas en función de los intereses políticos. 5. Referencias bibliográficas

\section{Más allá de los géneros de opinión}

La opción por un género condiciona la exposición del punto de vista. Los géneros de opinión orientan en un sentido concreto la actividad discursiva y la prediscursiva. Es decir, el ethos retórico que se impone el autor de este tipo de escrito parte de la convicción de que su enfoque se ha de manifestar de una forma evidente. Por el contra-

1 El presente artículo se ha desarrollado dentro del programa de investigación FFI2010-18514 (subprograma FILO), Subvencionado por el Ministerio de Ciencia y Tecnología titulado "Comunicación en la empresa y en las instituciones: mecanismos discursivos de gestión del conocimiento y persuasión social". 
rio, la noticia demanda de una presencia discreta del autor (latencia, si se quiere). Su actividad discursiva se inclina por un ethos en el que se transmita la apariencia de neutralidad. Hasta aquí podríamos diferenciar, en su concepción retórica, las consideraciones previas que los autores asumen; opinar, por un lado, o relatar los hechos, por el otro. Veremos que esto no es exactamente así, puesto que las formas de deslizar el punto de vista son variadas y el género no es una parcela infranqueable cuando se trata de influir al lector. Diríamos, con María Jesús Casals (2001: 197), que "la transgresión de los géneros y de sus exigencias éticas suele enmascarar intereses que se ocultan y la falta de verdad".

Es obvio que la consideración del modelo de lector subyace en estos escritos como elemento clave de modelización. Tras la idea de lector implícito se esconde la intención del autor de converger con los puntos de vista de su receptor, para convencerlo, o reforzar las opiniones que éste ya pudiera tener. De este modo aparece un primer condicionante de tipo contextual, de claras connotaciones sociológicas, que tiene que ver con los componentes ideológicos. Estos se hacen más evidentes en los escritos de opinión, más soslayados en las relaciones de hechos. Además, la línea editorial marca la trayectoria a seguir por los autores y un marco referencial de partida a la hora de considerar qué tipo de lectores tienen. En ese sentido la elección de un ethos retórico está ligada a determinados estereotipos (Maingueneau, 2002) que nos llevan a considerar al articulista como un personaje (López Pan, 2011) que se muestra, precisamente, a través de la elección retórica antes referida.

La diferencia de estilo en los géneros radica, a menudo, en la presencia explícita (argumentación) o la presencia latente (narración). Eso quiere decir que la opinión se muestra, en el primer caso, de una forma directa a través del discurso argumentativo. Por el contrario, en la narración funcionan otros mecanismos condicionantes de la interpretación. En ello juegan un papel importante mecanismos pragmáticos y de estilo como la elección léxica (eufemismos, disfemismos, marcas disfóricas, y otros sesgos semánticos) o la inferencia, porque suele ponerse en juego lo implícito.

El fenómeno atiende, por otra parte, a una concepción del Nuevo Periodismo que se parapeta tras la idea de borrar las fronteras de los géneros (Casals, 2001). Ahora bien, en ello podríamos ver, también, uno de los riesgos que se corren al franquear esas fronteras de la convención; principalmente que lo que debiera tener una apariencia objetiva pasa a ser una manifestación subjetiva y se cataloga de ese modo por parte del lector.

En la opinión, los sesgos ideológicos son más evidentes y manifiestos, como tendremos ocasión de comprobar. Para el caso de los géneros a priori más objetivos, el reportaje, la crónica y la noticia, observaremos diversas estrategias según las cuales llegamos a intuir una postura determinada por parte de ese narrador inadvertido de los hechos y que es, en realidad, quien introduce su punto de vista a través de una serie de elecciones tomadas entre diferentes opciones (estilo ligado a la pragmática). El mero hecho de presentar unos acontecimientos por delante de otros nos hace pensar ya en ese sentido de lo ético. Remitámonos a cómo titulan algunas noticias y qué tematizan.

Así titulaba el diario El Mundo una de las noticias: "Los seis detenidos en el Congreso, relacionados con el 15-M y los antisistema" (El Mundo,18-11-2011); también "Zapatero, ambiguo" (El Mundo, 20-5-2011). Lo que se titula, en el primer caso, es 
la implicación en un hecho delictivo de personas vinculadas con el movimiento, no se comenta si son una minoría del grupo o si son representantes autorizados de él. El término aparentemente neutro "relacionados" busca poner en relieve -puesto que aparece en titular- esos nexos. En el caso del segundo titular, la ambigüedad de la postura del entonces presidente del gobierno tiene relación con la voluntad de reflejar la carencia de un posicionamiento firme en contra del $15-\mathrm{M}$ por parte de Zapatero. El valor disfórico del término (ambiguo) aporta una serie de connotaciones que, desde la semántica en contexto, implica una valoración negativa. Se trata, por lo tanto, de vincular los hechos ocurridos alrededor del 15 de mayo de 2011 con la inacción del gobierno para reprimir el movimiento.

De ese modo, con la elección de determinados titulares, entendemos que el fenómeno del 15-M se presentó, por parte de la prensa, como un acto permitido por el gobierno, especialmente entre los días 18 y 22 de mayo, los inmediatamente anteriores a las elecciones, y los días posteriores. La elección del titular, o elección del tema, es clave en ese sentido y en esa cronología porque lo tendríamos que considerar como un establecimiento de agenda en época electoral. Sheafer y Weimann (2005: 349) hicieron un estudio sobre el proceder de los medios de comunicación en el período electoral de Israel. Nosotros lo aplicamos como una de nuestras claves interpretativas del tratamiento que la prensa prestó al 15-M durante el mes de mayo de 2011. Veamos, como caso ilustrativo, la portada de La Razón el día 20 de mayo de 2011. En la parte superior "Boicot a la democracia"; debajo, izquierda: "los indignados convocan una manifestación para la jornada de reflexión a pesar de la prohibición", derecha: "La web del PSOE sirvió de plataforma para las concentraciones previas al 15-M". En la parte baja "Almodóvar agita el fantasma del 13-M de 2004. El director se solidariza con los manifestantes desde la alfombra roja de Cannes".

Los mecanismos de relación cognitiva que quiere desencadenar esta última cita ilustran la voluntad del periódico de evitar que se repitiera lo vivido el 13 de marzo de 2004, cuando la gente salió a la calle para pedir que el gobierno de Aznar diese una explicación satisfactoria a la masacre de Atocha de dos días antes. Además, y en eso el sesgo ideológico de la noticia se muestra más sutil, hermana los sintagmas "se solidariza con los manifestantes" y "desde la alfombra roja" abriendo el camino a una inferencia según la cual el lujo, el glamour y la exquisitez del festival cinematográfico de la ciudad francesa (la elección de la expresión alfombra roja no es inocente) estarían en disonancia con los objetivos perseguidos por el 15-M y con la estética de los manifestantes. Esa conducción hacia la paradoja se fija en la figura de Almodóvar por haber sido uno de los personajes públicos que fue más allá de lo que pidieron los manifestantes del año 2004 y usó su proyección mediática para poner en entredicho la actuación del gobierno de Aznar.

Detrás de la concepción política que la derecha española tenía sobre lo sucedido en las elecciones de aquel año se escondía la consideración de que la victoria socialista había estado condicionada por aquella toma de las calles. Durante los días previos a la votación del 2011 el periódico citado gestiona aquellos precedentes y vincula lo pasado con la posibilidad de que se alterara lo que ellos consideraban "orden" o "democracia”. Es decir, La Razón presenta lo sucedido como alteración del curso de los 
acontecimientos que desde su óptica serían propios de una democracia (sin manifestaciones de ningún tipo).

\section{Lo dicho y su interpretación, o el malicioso uso de las comillas}

Más allá del uso de la palabra propia, que es lo que nos ocupaba en el caso anterior, nos podemos centrar en el de la palabra ajena. La utilización del discurso reportado, en versión directa o indirecta, se presta al empleo sesgado o intencionado de las palabras del otro. Veamos un claro ejemplo en el siguiente párrafo:

"Durante la rueda de prensa posterior al Consejo de Ministros, Rubalcaba se ha limitado a repetir en sus respuestas a las múltiples preguntas de los periodistas sobre este tema que «la Policía sabe lo que tiene que hacer» y que «tomará sus decisiones en función de lo que pase», sin aclarar si utilizará la fuerza contra las manifestantes." (El Mundo: 20/05/2011)

Con la expresión perifrástica "se ha limitado a repetir" se ofrece un punto de vista condicionado que va más allá de otras opciones como "ha repetido" "se ha limitado a decir". Los componentes semánticos de la "limitación" y de la "repetición" evidencian que el periodista ha querido presentar las palabras del entonces ministro como limitadas (poco amplias), insistentes, carentes de argumentos, usadas como parapeto. Por lo tanto, llegan ante la cita en estilo directo lo suficientemente condicionadas para que el lector tenga una idea previa a través de las calificaciones vertidas sobre las palabras de Rubalcaba. Aún más, presenta eso como respuesta a las "múltiples preguntas". De nuevo la calificación se sitúa del lado de la óptica de la voz que narra. Para el autor no fueron ni una ni dos, sino "múltiples", repetidas, las preguntas en el mismo sentido. Es decir, usa una estrategia de oposición cuantitativa: muchas preguntas, muchas veces la misma respuesta, una.

A ello suma, después del uso del estilo directo como si fuera un corte de voz, una nueva categorización de la intervención del vicepresidente: "sin aclarar". Impone una técnica de ruptura (aclarar si la policía usará la fuerza/ no aclararlo) en la que el segundo elemento sería el desvalorizado. Más adelante percute de nuevo en la desvalorización de éste al usar la anáfora referencial del siguiente modo "Ante la ambigüedad en estas respuestas". El uso del vocablo "ambigüedad" por el periodista calificará, filtrará el punto de vista discursivo. Observemos, por otro lado, la coincidencia léxica con el titular del mismo periódico citado en el epígrafe anterior: "Zapatero, ambiguo".

En una línea similar utilizaba el articulista de $A B C$ la cita en estilo directo de lo dicho por el Ministro del Interior, que está precedida por una remarca de subjetividad -argumento contrario expresado por "según palabras"- que se usa para contrastar con el incumplimiento de las resoluciones de las Juntas Electorales:

“Sin intervenir, porque, según palabras del ministro del Interior «la policía busca resolver problemas y no crear más problemas donde no los hay». Pese a lo dictado por la Junta Electoral Provincial de Madrid y, en segunda instancia por la Junta Electoral Central, al filo de la medianoche de ayer, todo sigue igual allí. (Martínez, 2011: 26) 
Los dos casos ahora expuestos contrastan con la profusión de citas que incluye el diario Público sobre la misma noticia:

"Rubalcaba ha mencionado tres principios básicos "«de los que no nos vamos a bajar»": oportunidad, congruencia y proporcionalidad. «Eso rige su actuación. Para que todos entiendan: la policía no crea un problema donde no lo hay, ni dos ni tres». Además ha asegurado que «la policía tiene muchos años de experiencia y se ha encontrado con todos los supuestos posibles». «Saben perfectamente qué hacer»

«Estas concentraciones se han desarrollado de manera pacífica, y eso es bueno y tranquilizador, y el Gobierno debe decir a los ciudadanos estén tranquilos», ha abundado para zanjar: «Este es un Estado que funciona bien»." (Águeda, 2011: 6)

Las respuestas, como vemos, son de diversa índole, en contraste con lo que indicaba la noticia del diario El Mundo, donde se decía que Rubalcaba "se había limitado a repetir". Ciertamente, se puede hacer una apreciación sobre el contenido de las respuestas -de hecho es lo que practicó el redactor de El Mundo- o se puede hacer una selección de las diversas respuestas ante la insistencia de los periodistas presentes en la rueda de prensa.

Veamos ahora un caso de transición del estilo indirecto al uso de las comillas, directo, para marcar la distancia respecto al enunciador de los calificativos: "Zapatero dice que los acampados «son pacíficos y merecen respeto»" (Sanz, 2011: 31). Con el titular de la noticia el autor consigue transferir las palabras del anterior Presidente del Gobierno marcando una distancia que se evidencia nada más iniciar la lectura del escrito:

"El PSOE llega al final de campaña con sensaciones contradictorias. Por un lado, practica surf sobre la ola de «indignación» en la esperanza de que despierte a su electorado y se convierta este domingo en un tsunami de votos para pasar por encima del PP." (Sanz, 2011:31)

Tenemos oportunidad de apreciar, por lo tanto, un acercamiento mayor a los géneros de opinión que en las citas anteriores. Se trata, en este ejemplo, de expresar o desvelar una intuición sobre la estrategia política seguida por el Partido Socialista Obrero Español en su campaña electoral. La metáfora surfista del autor nos lleva a intuir, de nuevo, la idea de la ambigüedad que determinados medios intentaron proyectar sobre la actuación del Gobierno Español en aquellos instantes. En este caso, además, se expresa de manera clara la opinión del articulista según la cual ese comportamiento equívoco tiene como intención recabar votos entre las filas de los indignados del 15-M.

Hasta aquí parece claro que una parte de la prensa española se posicionó en destacar la falta de decisión de Rodríguez Zapatero y de Alfredo Pérez Rubalcaba a la hora de reprimir y disolver a los concentrados en las plazas de España, particularmente a los acampados en la Puerta del Sol madrileña. Las marcas disfóricas que preceden al discurso citado así lo demuestran. La acusación directa, tal y como aparece en la última cita aportada, era que la izquierda pretendía sacar rendimiento electoral a la situación. En otros casos, como veremos, se acusará directamente a la izquierda política de estar detrás del movimiento. 
De todos modos, la estrategia electoral que se evidencia a través de la prensa no se vislumbra siempre en el mismo sentido y con el mismo signo. A continuación ofrecemos una panorámica diferente de los enfoques que fue adquiriendo en los periódicos este cúmulo de acontecimientos.

En el siguiente ejemplo, lejos de la sorpresa que nos pueda causar la lectura de la noticia -intentaremos darle una explicación ideológica a posteriori- vemos cómo se produce una enunciación en cascada. Se trata de las declaraciones del conocido Carlos Fabra, Presidente de la Diputación de Castellón en aquellos momentos:

"El dirigente popular se refirió a su hijo de 32 años, licenciado en Márketing y Publicidad, que habla italiano, inglés y alemán y está en paro, y aseguró que éste le dijo: «Papá, lo que piensan ellos lo pienso yo». «Tenemos muy poca confianza en el sistema y muy poca confianza en muchas cosas», señaló Fabra, quien sobre los jóvenes que se han concentrado en varias ciudades españolas dijo que «es gente que está desesperada y que han ido allí a decir "queremos trabajo"». En un país con «más de cinco millones de parados», Fabra ve en el movimiento 15-M «una respuesta bastante correcta por parte de la juventud», y también de «padres y madres que estaban pidiendo trabajo para sus hijos». (EFE, 2011)

En la noticia se destaca como titular, precisamente, aquello que dice Fabra, en estilo directo, con la voluntad de mostrar las palabras del dirigente del PP de Castellón como un guiño a la juventud, a los parados que se concentraban en las plazas de nuestras ciudades. Si nos fijamos en la alternancia del discurso directo con el indirecto intuimos que la voluntad de mostrar las aptitudes del hijo del Presidente del PP castellonense se filtra a través de la voz narrativa con un "se refirió a su hijo de 32 años, [...]que habla italiano, inglés y alemán y está en paro, y aseguró que éste le dijo ${ }^{2}$ : [...] El estilo directo, de hecho, está usado como enunciación en eco, dentro del estilo indirecto ("aseguró que"). Puestos a aplicar criterios de lógica en el enunciado, nos encontramos con que la voz narrativa (locutor que usa el EI), se vale de un segundo locutor (L2), que a su vez asume la voz de un L3 y la cita con una voluntad de resultar más verosímil a través del uso del estilo directo. Es decir, es el señor Carlos Fabra quien cita lo que dice su hijo, Borja Fabra, cosa que constituye un filtro, un distanciamiento de voces evidente entre el autor del escrito y el hijo del presidente local del PP.

Visto así, la cascada de locutores (3) sirve de estrategia para mostrar lejanía entre el primero y el tercero. En ningún momento se afirma que aquello que dice L3 se haya contrastado, grabado o transcrito, se trata del uso de unas presuntas palabras de Borja Fabra, al que su padre, Carlos, atribuye una serie de virtudes, de preparación (habla italiano, inglés y alemán). Reformulada y sintetizada, la noticia sería que Fabra dijo que su hijo Borja habla italiano, inglés y alemán; que su hijo Borja, que está en paro, dijo: "Papá, lo que piensan ellos lo pienso yo". Es decir, Fabra identifica el descontento social del 15-M (por el paro) con la situación de su hijo, al que califica como altamente preparado.

Evidentemente, la inferencia que se desprende es que el entonces presidente de la Diputación de Castellón intentaba buscar el nexo de unión del descontento social (ju-

2 La cursiva es nuestra. 
ventud preparada en paro) con la situación sociopolítica española de la cual se culpabilizaba al gobierno del Estado. Quizás por eso "se refirió a su hijo" antepone una especie de lente que condiciona la óptica del lector, que unido al "aseguró que" sirve para distanciar al locutor originario $(K)$ del período entrecomillado. En definitiva, algo que se podría resumir en un "según Fabra, su hijo le dijo: [...]" pasa por "se refirió", para acabar con la sentencia conclusiva del propio Fabra: "es gente que está desesperada y que han ido allí a decir «queremos trabajo»".

Queda abierta la puerta a dudar sobre la veracidad de lo dicho por el hijo del conocido líder político, puesto que esta enunciación en doble eco quiere focalizarse más sobre el guiño que el dirigente político hace a los indignados, a los parados, desde el interés político en una estrategia de uso de la figura del hijo como fuente argumental (Lo Cascio, 1998) quizás una estrategia de particularización (Perelman/Olbrechts-Tyteca, 1989) contra el gobierno. La valoración de la conclusión del propio Fabra nos sirve para consolidar esta idea, puesto que se intenta ligar la situación sociopolítica española con el fenómeno. Es decir, etiquetarlo desde el descontento con el Gobierno del Estado Español en aquellos momentos.

La estrategia consistiría en que el Presidente del PP de Castellón pone como ejemplo de persona con motivos para indignarse a su propio hijo. La noticia, por lo tanto, filtra la voluntad del señor Fabra de incidir en el descontento con la política gubernamental, como un guiño a la indignación de los jóvenes. Curiosamente queda bastante claro el interés del político en sacar rédito electoral del movimiento a través de sus declaraciones de simpatía hacia el 15-M, cosa de la cual, paradójicamente, algunos periódicos estaban acusando al gobierno Zapatero. Carlos Fabra asumía, así, la voz de padre de un parado descontento sin dejar de acceder a los medios de comunicación en su calidad de líder de un partido político.

Si fuéramos más allá de la consideración de las palabras aisladamente y nos adentráramos en la posición que ocupan, nos daríamos cuenta de que el estilo indirecto ha sido la antesala del estilo directo. En los ejemplos se ha jugado a colocar la voz del periodista como filtro antes que la voz del personaje.

Si nos preguntamos por el significado de este orden, cabría la posibilidad de entender que la distribución de las palabras tiene un sentido bien evidente. El discurso directo está flanqueado por un discurso indirecto, antepuesto y postpuesto, que se aleja de un estado puro de la noticia -la pureza la representaría, en este caso, la transcripción de la entrevista o rueda de prensa-, puesto que los calificativos adquieren un valor semántico que manifiesta el punto de vista del autor. Sirven a la estrategia de crear advertencias orientativas en la presentación de lo citado.

\section{Dimensión pragmaestilística del discurso}

En los ejemplos anteriores -sobre todo en el segundo- hemos contemplado cómo se han usado adjetivos y una nominalización anafórica para inclinar las ópticas del lector sobre los hechos referidos. Las elecciones léxicas nos ayudan, a menudo, a manifestar de una manera más flagrante el punto de vista. Los marcos cognitivos a los que nos remiten determinadas expresiones dan una idea clara de cómo ven algunos columnistas el fenómeno del 15-M. 
La búsqueda de las causas del movimiento y el intento de responsabilizar al gobierno del momento se unifican como estrategia en determinado tipo de prensa escrita. Si la mayoría de estos medios lo hacían en los géneros aparentemente neutros, en los de opinión entran a marcar directamente como responsable al Gobierno de la nación. Así, observando un editorial del diario $A B C$ de aquellos días, nos encontramos con una reflexión metadiscursiva curiosa. El editorial alude a su análisis como objetivo, mientras que usa una metáfora para calificar la postura de los miembros del 15M como subjetiva y poco definida. Dice así:

"Hay razones para el desencanto ante la realidad de la crisis, pero, objetivamente el responsable es un gobierno de izquierdas y no «el sistema», cajón de sastre ${ }^{3}$ en el que los manifestantes depositan sus reproches" (ABC, 19 de mayo de 2011, p. 4)

Parece claro que aquello que intentaba ofrecer desde sus editoriales la prensa más cercana al Partido Popular era que la causa del descontento estaba generada por la situación a la que nos había llevado el gobierno socialista. Eso, sin embargo, entraba en contradicción con los vínculos que se querían establecer entre los manifestantes y la izquierda. Es decir, se usaba la ligazón entre izquierda y movimiento cuando se trataba de mostrarlo en su carácter conflictivo y de denostarlo por su aliño. Sin embargo, cuando se trataba de analizar las razones del descontento, se buscaba una relación causal en el gobierno de izquierdas. Esa paradoja se iría diluyendo después del día 22 de mayo de 2011, cuando estratégicamente ya no interesaba culpabilizar al gobierno como causante de las manifestaciones. En ese instante parece que el estilo periodístico se orientó hacia la presentación de un 15-M más cercano a la izquierda, visto desde la perspectiva de una óptica conservadora más próxima a tópicos sociales que convendría desgranar.

A continuación ofrecemos alguna de las apreciaciones más sustanciosas que sobre el 15-M han hecho algunos opinadores desde las tribunas de sus periódicos. En respuesta a la presunta manipulación de una portada por parte de La Razón, el periodista Ignacio Villa dedicaba su escrito a expresar, a través de un estilo denotativo bastante evidente, lo que habíamos calificado como postura tomada por parte de los sectores mediáticos cercanos a la derecha. Se trataba de ligar el movimiento 15-M a las fuerzas de la izquierda política, tanto parlamentaria como extraparlamentaria. Villa contesta a las acusaciones de manipulación con una serie de calificaciones en las que la estrategia argumental consiste en situarse en la posición de víctima de las iras de los sectores progresistas. Según el autor, la izquierda no mantiene una actitud crítica y sosegada, él es quien se constituye en portavoz de esos valores positivos. Las marcas disfóricas sobre la izquierda ("inquisidora, rabiosa y desorientada") contrastan con los valores semánticos que atribuye a su posición y a la de su periódico (imagen clara y nítida; actitud crítica y sosegada):

"La portada de LA RAZÓN era una imagen panorámica de la plaza de Neptuno, clara y nítida. Otra cosa diferente es que la izquierda, inquisidora rabiosa y desorientada, ahora

3 La cursiva es nuestra. 
se dedique atacar a los medios que mantienen una actitud crítica y sosegada sobre la actualidad. Es una pena. Y flaco servicio hacen al 15-M, que desde luego no tiene carácter redentor de nada, ni de nadie." (Villa, 2011)

Observamos que la descalificación del movimiento tomaba, en los días inmediatamente anteriores y posteriores a la celebración de la jornada electoral, recordémoslo, un cariz evidente. Al artículo referido podemos sumar la contribución de Alfonso Ussía, en la que la retahíla de calificaciones se une a la voluntad de deslegitimación por irrelevancia. Para el autor, además de escasos en número, los manifestantes eran gente poco dada a la higiene, véase el eufemismo usado con voluntad satírica:

"Lo cierto es que en Madrid fueron pocos los manifestantes presumiblemente indignados. Uniformidad antisistema, estudiado desaliño marginal y espesura corporal acentuada por el calor. (Ussía, 2011)

Tanto en el caso de Villa como en el de Ussía tenemos ejemplos de categorización a través de las elecciones léxicas que practican. El movimiento, para Villa, es de izquierdas y, por lo tanto, inquisitorial, rabioso y desorientado. Frente a ello contrapone los valores de moderación que se arroga, para él y para su periódico. En el caso de Ussía, la sátira viene definida por la voluntad de ataque del propio recurso, centrándose en la imagen que quiere dar de los concentrados: con pose estudiada y estética de corte marginal, además de sucios.

Resulta evidente que los casos de géneros de opinión -como en los dos ejemplos citados - el uso de adjetivaciones con una intención perlocutiva resulta evidente. En las noticias y en los reportajes el uso de las comillas responde, como decíamos, a una estrategia discursiva más elaborada. El sentido que se da al discurso reportado está en función de los intereses para su aprovechamiento en la construcción de la óptica sobre los hechos. Como veíamos, cuando se trataba de ofrecer una perspectiva de ambigüedad los períodos entrecomillados escaseaban y, además, venían acompañados de modalizadores con sesgos semánticos evidentes.

Por el contrario, cuando se pretendía ofrecer una visión muy distanciada sobre una rueda de prensa, en la que quedara claro que las palabras citadas no tenían nada que ver con el autor de la redacción -el caso del señor Fabra- y se incluían mecanismos lingüísticos (oraciones completivas) que propiciaran ese alejamiento. A ello cabía sumar el uso de la enunciación en doble eco para acrecentar ese punto de vista.

\section{Conclusión: estrategias discursivas en función de los intereses políticos}

De las formas de presentar verbalmente estos hechos, deberíamos sacar una serie de conclusiones dirigidas a relacionar los discursos analizados con los vínculos sociales y políticos. El uso que se hizo del 15-M pasó por varias etapas. En un principio se dieron pocas noticias. Más adelante hubo un intento de simpatizar, desde la estrategia política, a la vista de unas elecciones que se aproximaban y de las resonancias que el movimiento pudiera tener. Aquellos articulistas que presentaban el movimiento como reacción al paro en nuestra sociedad, lo hacían desde la óptica de los que buscan las causas en la acción de gobierno. Pero cabría matizar si esa acción de gobierno res- 
ponsabilizaba directamente al del Estado o podría buscar la responsabilidad de determinados ejecutivos autonómicos y locales.

La cronología seguida en la presentación de los acontecimientos en periódicos afines coincide con la perspectiva que los partidos políticos intentaron ofrecer. De hecho, si nos fijamos en cómo presenta el diario Público la posición de Esperanza Aguirre, presidenta de la Comunidad de Madrid, vemos la coincidencia estratégica a la que hemos aludido cuando la prensa conservadora se posicionó por la disolución de los concentrados. Las razones las esgrime de una manera bastante clara porque cita expresamente lo ocurrido en marzo de 2004:

"Sobre los indignados, dijo [Esperanza Aguirre] tratarse de un grupo «muy heterogéneo» con motivos «muy justificados». Y mostró su preocupación por el efecto que pueda tener en la jornada de reflexión la prohibición de la Junta. «La experiencia que tenemos en el PP de la última jornada de reflexión de las generales fue realmente dramática», aseguró en declaraciones a Onda Cero recogidas por Ep. (González, 2011)

En la medida en que hemos seguido las posiciones adoptadas en los géneros teóricamente imparciales por parte de los periódicos afines al Partido Popular entendemos que se buscaba justificar el 15-M en el mismo sentido político -indignación contra el ejecutivo de Zapatero- y que los temores a repetir los episodios de 2004 centraron los esfuerzos de La Razón, ABC, El Mundo, ... en la presión mediática para que la policía disolviera a los acampados.

Leyendo el periódico Levante-El Mercantil Valenciano de Valencia, podemos llegar a entender que la indignación no tenía un único causante. De hecho, la selección de acontecimientos, la "inventio" retórica propiciada por la redacción del periódico el día 19 presenta como corresponsables de la situación a la Generalitat Valenciana y al Ayuntamiento de Valencia.

"Asimismo, los manifestantes han pedido que las personas que ocupen la presidencia de la Generalitat y la alcaldía de Valencia tras el 22-M den explicaciones ante la asamblea de la situación financiera de la Generalitat y el Ayuntamiento, así como de los costes reales" y beneficios de los denominados "grandes eventos". (EFE, 2011b).

Obviamos decir que en otro tipo de prensa esto se silenció. Además, todo lo anteriormente visto nos lleva a considerar que aquello que había servido para la culpabilización del gobierno de Zapatero dejó de ser útil para un sector después de las elecciones autonómicas y municipales, puesto que había alentado la búsqueda de la causa del descontento en el gobierno central y su finalidad ya se había cumplido. Así se pasó a pedir la retirada de los concentrados basándose en el argumento de la necesidad de respeto a la jornada de reflexión y a la decisión de instancias judiciales (JEL y JEC). En ese momento el paso estratégico fue poner al gobierno en el brete de tomar la decisión de disolver las concentraciones (achacarle ambigüedad). Si lo hacía, el gobierno se arriesgaba a producir un conflicto violento; si no lo hacía, la prensa afín al Partido Popular lo calificaría de tibio, indeciso y de no respetar las resoluciones de las Juntas Electorales. 
Además, a ello se podía añadir un argumento de suma: la connivencia del PSOE con los concentrados, cosa que se explotó mayormente a posteriori, una vez celebrados los comicios autonómicos y locales.

En el presente artículo hemos desarrollado un análisis mediante el cual pretendíamos presentar los vínculos entre el estilo periodístico, las elecciones de signos (de puntuación, léxicosemánticos, sintácticos...) para enfocar los hechos según un punto de vista que es el que se trata de trasladar a los lectores. Es la intención, por lo tanto, de condicionar la opinión de los lectores, mediatizarla a través de una estrategia que coincide en un amplio sector de la prensa, en un sentido y en otro. Desde la elección de un enfoque referencial previo (fixed frame, en terminología de G. Lakoff) para presentar los hechos, se diagnostica un proceso de reelaboración en función de los criterios sociopolíticos, a veces coincidentes con estrategias políticas precocinadas. Así se ofrecen a la sociedad bajo una óptica que permite, una vez gestionado el discurso de presentación del movimiento 15-M, sumirlos en tópicos con apelativos del tenor de "perro-flautas" u otros calificativos empleados en artículos de opinión de la prensa española.

\section{Referencias bibliográficas}

ÁGUEDA, Pedro (2011): "El gobierno descarta desalojar a los indignados", Público, 20-05-2011, p. 6

CASALS CARRO, María Jesús (2001): "La narrativa periodística o la retórica de la realidad construida". Estudios sobre el Mensaje Periodístico, vol.7, pp. 195-219. Madrid, Servicio de Publicaciones de la Universidad Complutense

EFE (2011a): http://www.levante-emv.com/comunitat-valenciana/2011/05/19/fabrami-hijo-dice-papa-piensan-pienso/808238.html. [19-07-2011]

EFE (2011b): http://www.levante-emv.com/comunitat-valenciana/2011/05/19/protestas-dejan-oir/808390.html [19-07-2011]

GONZÁLEZ, Yolanda (2011): http://www.publico.es/espana/377115/la-izquierda-semuestra-complice-con-la-movilizacion-de-la-puerta-del-sol-elecciones2011 [1905-2011]

LO CASCIO, Vicenzo (1998): Gramática de la argumentación: estrategias y estructuras. Madrid, Alianza Editorial.

LOPEZ PAN, Fernando (2011): "El articulista-personaje» como estrategia retórica en las columnas personales o literarias". Anàlisi, vol. 41, pp. 47-68.

MAINGUENEAU, Dominique (2002):"Problèmes d'ethos", en Pratiques, 113/114, 55-68.

MARTÍNEZ, Álvaro (2011): “Los «indignados» se acantonan”, ABC 20-5-2011, p. 26.

PERELMAN, Chaïm, \& OLBRECHTS-TYTECA, Lucie (1989): Tratado de la argumentación. La nueva retórica. Madrid, Gredos.

SANZ, Gabriel (2011): "Zapatero dice que los acampados «son pacíficos y merecen respeto»" $A B C, 20-05-2011$, p. 31 
SHEAFER, Tamir and WEIMANN, Gabriel (2005): “Agenda Building, Agenda Setting, Priming, Individual Voting Intentions, and the Aggregate Results: an analysis of four Israeli elections", Journal of Communication 55(2), pp. 347-65.

USSÍA, Alfonso (2011): http://www.larazon.es/noticia/9632-pocos [20 de julio de 2011]

VILLA, Ignacio (2011): http://www.larazon.es/noticia/3980-la-izquierda-inquisidora [19 de julio de 2011] 